\title{
Free Group Rings and Derived Functors
}

\author{
Roman Mikhailov*and Inder Bir S. Passi
}

\begin{abstract}
An approach to identify the normal subgroups determined by ideals in free group rings with the help of the derived functors of non-additive functors is explored. A similar approach, i.e., via derived functors, for computing limits of functors from the category of free presentations to the category of abelian groups, arising from commutator structure of free groups, is also discussed.
\end{abstract}

2010 Mathematics Subject Classification. 18A30, 18E25, 20C05, 20C07

Keywords. Group rings, derived functors, generalized dimension subgroups.

\section{Introduction}

Let $F$ be a free group and $\mathbb{Z}[F]$ its integral group ring. In the theory of group rings a repeatedly occurring theme is the identification of normal $\operatorname{subgroups} D(F, \mathfrak{a}):=$ $F \cap(1+\mathfrak{a})$ determined by two-sided ideals $\mathfrak{a}$ in $\mathbb{Z}[F]$ (see, e.g., [4], [10], [13]). It is often the case that a certain normal subgroup $N$, say, is easily seen to be contained in $D(F, \mathfrak{a})$, and computing the quotient $D(F, \mathfrak{a}) / N$ usually becomes a challenging problem. A classical instance of this phenomenon is the computation of the so-called dimension quotients $D\left(F, \mathbf{r}+\mathbf{f}^{n}\right) / R \gamma_{n}(F), n \geq 1$, for a group $G$ with free presentation $G \cong F / R$, where $\mathbf{r}$ denotes the two-sided ideal of the group ring $\mathbb{Z}[F]$ generated by $R-1$, and $\gamma_{n}(F)$ is the $n$th term of the lower central series of $F$. More generally, if $R_{1}, R_{2}, \ldots, R_{n}$ are normal subgroups of $F$ and $\mathfrak{a}$ is a sum of certain products $\mathbf{r}_{i_{1}} \ldots \mathbf{r}_{i_{s}}$, then the identification of $D(F, \mathfrak{a})$ is usually an intractable problem. However, it has recently been noticed that derived functors of non-additive functors in the sense of Dold-Puppe [2] can be a useful tool for investigations in this area; for, the quotient $D(F, \mathfrak{a}) / N$ can sometime turn out to have interesting homological or homotopical interpretation. To mention an instance, let $R$ be a normal subgroup of a free group $F$. It is well-known (see [4]) that $D\left(F, \mathbf{f}^{n}\right)=\gamma_{n}(F)$, for all $n \geq 1$, and $D(F, \mathbf{f r})=\gamma_{2}(R)$. Surprisingly, it turns out that $D\left(F, \mathbf{f}^{3}+\mathbf{f r}\right)$ is related to the first derived functor of the second symmetric power functor: $L_{1} \mathrm{SP}^{2}\left(F /\left(\gamma_{2}(F) R\right)\right)$ [5. The purpose of the present study is to continue further our work in [1] on the relationship between free group rings and derived functors of non-additive functors. In another direction, we extend our results in 11 on the connection between derived functors in the sense of Dold-

${ }^{*}$ The first author acknowledges Saint-Petersburg State University for a research grant N 6.37.208.2016. This paper was completed during the visit of the first author to Indian Institute of Science Education and Reseach Mohali; he wishes to express his gratitude to the Institute for its warm hospitality. 
Puppe and limits of functors from the category of free presentations of groups to the category of abelian groups.

Given a polynomial endofunctor $\mathcal{F}$ of degree $n$ (see 3 ) on the category of abelian groups, say, for example, the $n$th symmetric power $\mathrm{SP}^{n}$, the $n$th Lie power $\mathfrak{L}^{n}$, the $n$th super-Lie power $\mathfrak{L}_{s}^{n}$, or certain Schur functor, it has (in general, nonzero) derived functors $L_{i} \mathcal{F}, i=0,1,2, \ldots$, in the sense of Dold-Puppe 2. It turns out that the zeroth and the $(n-1)$ st derived functors $L_{0} \mathcal{F}, L_{n-1} \mathcal{F}$ usually are the simplest ones to compute, and, in general they have a simple combinatorial description. It is naturally to be expected that they appear in our analysis. In general, intermediate derived functors have a complicated structure, their appearance in our study is indeed rather unexpected.

We begin by recalling, in Section 2, a needed basic fact about free group rings. In Section 3 we develop the results on derived functors of certain functors which help establish a connection between derived functors and subgroups determined by ideals in free group rings. The main results of this paper are in Section 4, where we exhibit several quotients of subgroups determined by ideals in free group rings in terms of derived functors. To mention here just one of our results, Theorem 4.2 states that if $1 \rightarrow R \rightarrow F \rightarrow G \rightarrow 1$ is a free presentation of a group $G$, and $S$ is the commutator subgroup $[R, F]$, then there are natural isomorphisms

$$
\begin{aligned}
& \frac{F \cap(1+\mathbf{r f r}+\mathbf{s r})}{\gamma_{2}(S) \gamma_{3}(R)} \cong L_{1} \mathrm{SP}^{2}\left(H_{2}(G)\right), \\
& \frac{F \cap\left(1+\mathbf{r f r}+\mathbf{r}^{2} \mathbf{f}\right)}{\gamma_{2}(S) \gamma_{3}(R)} \cong L_{1} \mathrm{SP}^{2}\left(H_{2}(G)\right), \\
& \frac{F \cap\left(1+\mathbf{s}^{2} \mathbf{r}+\mathbf{r}^{2} \mathbf{f r}\right)}{\gamma_{3}(S) \gamma_{4}(R)} \cong L_{2} \mathfrak{L}_{s}^{3}\left(H_{2}(G)\right),
\end{aligned}
$$

where $H_{2}(G)$ is the second integral homology group of the group $G$. Finally, in Section 5 , we give a number of identifications of the limits of functors, on the category of free presentations of groups, as derived functors. Again, to mention just one result, we prove in Theorem [5.1 that if a group $G \cong F / R$ is 2-torsion-free, then

$$
\lim _{\longleftarrow} \frac{R^{\prime \prime}}{\gamma_{2}\left(\left[\gamma_{2}(R), F\right]\right) \gamma_{3}\left(R^{\prime}\right)} \cong L_{1} \mathrm{SP}^{2}\left(H_{4}(G, \mathbb{Z} / 2)\right),
$$

where $R^{\prime}, R^{\prime \prime}$ are respectively the first and the second derived subgroups of $R$.

For background on derived functors of non-additive functors, we refer the reader to [1] and [2], and, for free groups rings, to [4].

\section{Preliminaries}

For a normal subgroup $H$ of a group $G, \mathbf{h}$ denotes the two-sided ideal $(H-1) \mathbb{Z}[G]$ of the integral group ring $\mathbb{Z}[G]$. We denote by $\gamma_{n}(G), n \geq 1$, the $n$th term in the 
lower central series of $G$ defined inductively by setting

$$
\begin{aligned}
G=\gamma_{1}(G), \quad \gamma_{n+1}(G)=\left[G, \gamma_{n}(G)\right] & = \\
\langle[x, y] & :=x^{-1} y^{-1} x y\left|x \in G, y \in \gamma_{n}(G)\right\rangle, n \geq 1
\end{aligned}
$$

For notational convenience, we also denote the derived subgroup $\gamma_{2}(G)$ by $G^{\prime}$.

Let $F$ be a free group, $\mathfrak{b} \subset \mathfrak{a}$ and $\mathfrak{d} \subset \mathfrak{c}$ ideals of $\mathbb{Z}[F]$ such that

$$
\operatorname{Tor}(\mathbb{Z}[F] / \mathfrak{a}, \mathbb{Z}[F] / \mathfrak{c})=0
$$

where Tor $=\operatorname{Tor}_{1}^{\mathbb{Z}}$. Then the map $(x, y) \mapsto x y, x \in \mathfrak{a}, y \in \mathfrak{c}$, induces an isomorphism ([6], Lemma 4.9.)

$$
(\mathfrak{a} / \mathfrak{b}) \otimes_{\mathbb{Z}[F]}(\mathfrak{c} / \mathfrak{d}) \cong \frac{\mathfrak{a} \mathfrak{c}}{\mathfrak{b} \mathfrak{c}+\mathfrak{a} \mathfrak{d}}
$$

\section{Derived functors}

For a functor $T: \mathcal{C} \rightarrow \mathcal{A}$ from an abelian catefory $\mathcal{C}$ to the category $\mathcal{A}$ of abelian groups, $L_{p} T$ denotes the $p$ th derived functor of $T$ at level 0 , i.e., the functor $L_{p} T(-, 0)$ in the notation of Dold-Puppe [2]. Recall that the functor $T: \mathcal{C} \rightarrow \mathcal{A}$ is said to be a polynomial functor of degree $\leq n$ if the $(n+1)$ st cross-effect $T^{[n+1]}$ is zero $[3$.

3.1. Quadratic functors. Let $Q$ be a free abelian group, and $U$ a subgroup of $Q$. Then we have the following commutative diagram with exact rows and columns (see [1], [8]):

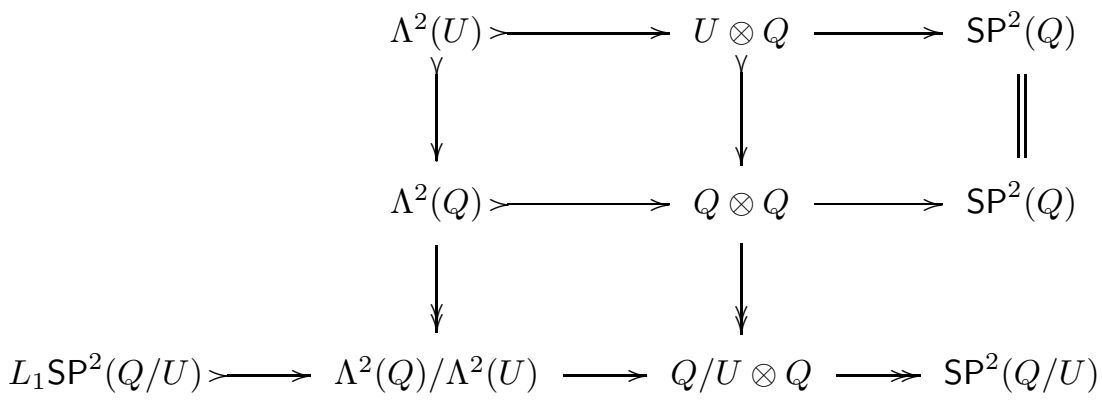

where $\Lambda^{2}$ and $\mathrm{SP}^{2}$ are the exterior square and the symmetric square endofunctors respectively on the category $\mathcal{A}$, and the homomorphisms are the natural maps induced by the inclusion $U \subset Q$ or the projection $Q \rightarrow Q / U$. Of particular interest to us is the lower 4-term exact sequence; as such we display it separately for later reference: 


$$
0 \rightarrow L_{1} \mathrm{SP}^{2}(Q / U) \rightarrow \Lambda^{2}(Q) / \Lambda^{2}(U) \rightarrow Q / U \otimes Q \rightarrow \mathrm{SP}^{2}(Q / U) \rightarrow 0
$$

The following result, which is a generalization of the corresponding result in 8], plays a crucial role in establishing a connection between subgroups determined by ideals in free group rings and derived functors.

Theorem 3.1. Let $E$ be an abelian group, and $I$ a subgroup of $E$. The first homology of the Koszul-type complex

$$
\Lambda^{2}(I) \rightarrow I \otimes E \rightarrow \mathrm{SP}^{2}(E),
$$

where the two homomorphisms are the natural maps induced by the inclusion $I \subseteq$ $E$, is naturally isomorphic to

$$
\text { Coker }\left\{L_{1} \mathrm{SP}^{2}(E) \rightarrow L_{1} \mathrm{SP}^{2}(E / I)\right\}
$$

Proof. Let $Q$ be a free abelian group with subgroups $U \subset V$ such that

$$
Q / U=E, \quad V / U=I
$$

Consider the following diagram

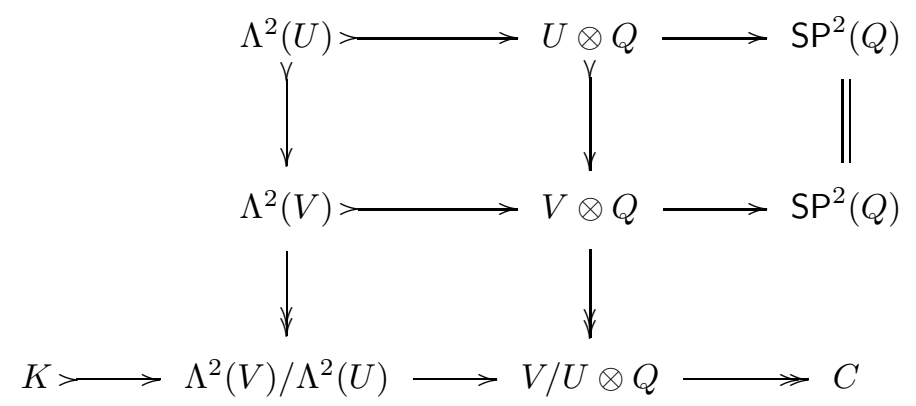

resulting from the natural map between representatives

$$
L \operatorname{SP}^{2}(E): \quad \Lambda^{2}(U) \rightleftharpoons U \otimes Q \longrightarrow \operatorname{SP}^{2}(Q)
$$

and

$$
L \operatorname{SP}^{2}(E / I): \quad \Lambda^{2}(V) \longrightarrow V \otimes Q \longrightarrow \mathrm{SP}^{2}(Q)
$$

of $L_{1} \mathrm{SP}^{2}(E)$ and $L_{1} \mathrm{SP}^{2}(E / I)$ respectively in the derived category of $\mathcal{A}$, where

$$
K=\operatorname{Ker}\left\{L_{1} \mathrm{SP}^{2}(E) \rightarrow L_{1} \mathrm{SP}^{2}(E / I)\right\}
$$


and $C$ lives in the exact sequence

$$
L_{1} \mathrm{SP}^{2}(E) \rightarrow L_{1} \mathrm{SP}^{2}(E / I) \rightarrow C \rightarrow \mathrm{SP}^{2}(E) \rightarrow \mathrm{SP}^{2}(E / I)
$$

as can be seen by easy diagram chasing. We assert that

$$
C=I \otimes E / \operatorname{Im}\left(\Lambda^{2}(I) \rightarrow I \otimes E\right)
$$

To see this, let us present $Q$ as $F / F^{\prime}$ with $F$ a free group and $F^{\prime}$ its derived subgroup, and let $R \subset S$ be normal subgroups of $F$ such that

$$
U=R / F^{\prime}, \quad V=S / F^{\prime} .
$$

With our notation for ideals in group rings induced by normal subgroups, we have, in view of (2.1), natural isomorphisms

$$
I \otimes Q \cong \frac{\mathbf{s}+\mathbf{f}^{2}}{\mathbf{r}+\mathbf{f}^{2}} \otimes \frac{\mathbf{f}}{\mathbf{f}^{2}} \cong \frac{\mathbf{s f}+\mathbf{f}^{3}}{\mathbf{r f}+\mathbf{f}^{3}}
$$

Therefore, there is a natural isomorphism

$$
C \cong \frac{\mathbf{s f}+\mathbf{f}^{3}}{\left(S^{\prime}-1\right)+\mathbf{r f}+\mathbf{f}^{3}}
$$

On the other hand,

$$
I \otimes E \cong \frac{\mathbf{s}+\mathbf{f}^{2}}{\mathbf{r}+\mathbf{f}^{2}} \otimes \frac{\mathbf{f}}{\mathbf{r}+\mathbf{f}^{2}} \cong \frac{\mathbf{s} \mathbf{f}+\mathbf{f}^{3}}{\mathbf{r f}+\mathbf{s r}+\mathbf{f}^{3}}
$$

Now observe that

$$
I \otimes E / \operatorname{Im}\left(\Lambda^{2}(I) \rightarrow I \otimes E\right) \cong \frac{\mathbf{s f}+\mathbf{f}^{3}}{\left(S^{\prime}-1\right)+\mathbf{r f}+\mathbf{s r}+\mathbf{f}^{3}}=\frac{\mathbf{s f}+\mathbf{f}^{3}}{\left(S^{\prime}-1\right)+\mathbf{r f}+\mathbf{f}^{3}} \cong C
$$

and thus the isomorphism (3.4) is proved. Consequently the assertion in the Theorem follows from the exact sequence (3.3).

Given a subgroup $I$ of an abelian group $E$, let $\Lambda^{2}(I)$ denote the image of the map $\Lambda^{2}(I) \rightarrow \Lambda^{2}(E)$ induced by the natural inclusion map $I \hookrightarrow E$.

Theorem 3.2. If $E$ is an abelian group, $I$ a subgroup of $E$ and

$$
\operatorname{Tor}(E / I, E) \rightarrow L_{1} \mathrm{SP}^{2}(E / I)
$$

the composition of the two natural maps

$$
\operatorname{Tor}(E / I, E) \rightarrow \operatorname{Tor}(E / I, E / I) \rightarrow L_{1} \mathrm{SP}^{2}(E / I),
$$

then there is a natural isomorphism

$$
\operatorname{Coker}\left\{\operatorname{Tor}(E / I, E) \rightarrow L_{1} \mathrm{SP}^{2}(E / I)\right\} \cong \operatorname{Ker}\left\{\Lambda^{2}(E) / \underline{\Lambda^{2}(I)} \rightarrow E / I \otimes E\right\}
$$


Proof. Consider the following commutative diagram with exact columns:

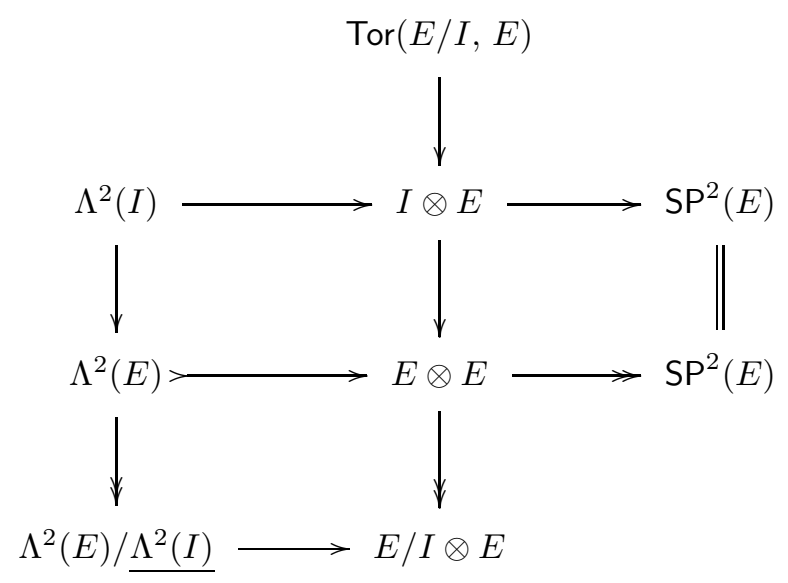

Note that the middle horizontal sequence is exact. The homology exact sequence together with Theorem 3.1 implies that there is a natural exact sequence

$\operatorname{Tor}(E / I, E) \rightarrow \operatorname{Coker}\left\{L_{1} \mathrm{SP}^{2}(E) \rightarrow L_{1} \mathrm{SP}^{2}(E / I)\right\} \rightarrow \operatorname{Ker}\left\{\Lambda^{2}(E) / \underline{\Lambda^{2}(I)} \rightarrow E / I \otimes E\right\}$.

However,

$$
\operatorname{Im}\left\{L_{1} \mathrm{SP}^{2}(E) \rightarrow L_{1} \mathrm{SP}^{2}(E / I)\right\} \subseteq \operatorname{Im}\left\{\operatorname{Tor}(E / I, E) \rightarrow L_{1} \mathrm{SP}^{2}(E / I)\right\}
$$

and we thus obtain the asserted isomorphism.

3.2. Cubic functors. For an abelian group $A$, recall that $\mathfrak{L}_{s}^{3}(A)$, the third super Lie functor evaluated at $A$ [1], is by definition the abelian group generated by brackets $\{a, b, c\}, a, b, c \in A$, which are additive in each variable, and satisfy the following defining relations:

$$
\begin{aligned}
& \{a, b, c\}=\{b, a, c\}, \\
& \{a, b, c\}+\{c, a, b\}+\{b, c, a\}=0 .
\end{aligned}
$$

Let $Q$ be a free abelian group, and $U$ a subgroup of $Q$. We note that the following diagram

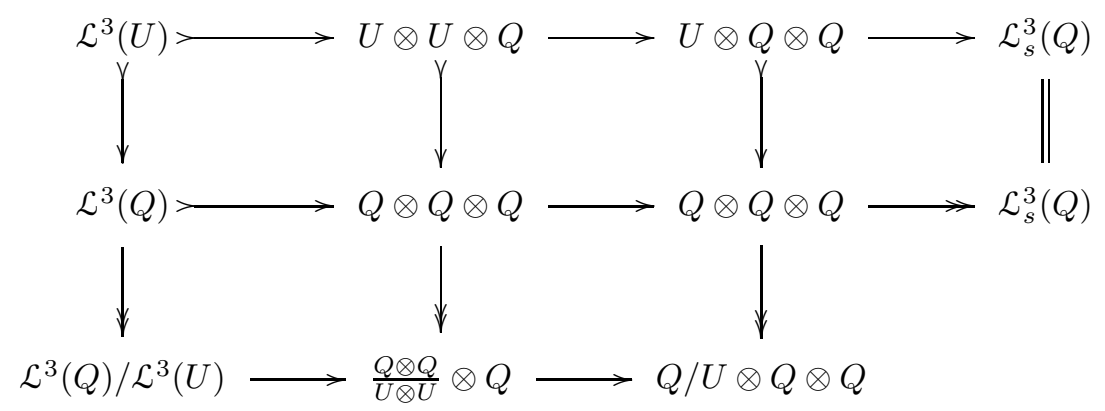


yields the natural exact sequence

$$
0 \rightarrow L_{2} \mathfrak{L}_{s}^{3}(Q / U) \rightarrow \mathcal{L}^{3}(Q) / \mathcal{L}^{3}(U) \rightarrow \frac{Q \otimes Q}{U \otimes U} \otimes Q
$$

3.3. Metabelian Lie functor. Let $1 \rightarrow R \rightarrow F \rightarrow G \rightarrow 1$ be a free presentation of a group $G$. Let $H_{a b}$ denote the abelianization $H / H^{\prime}$ of the group $H$. Again, it may be noted that the following diagram

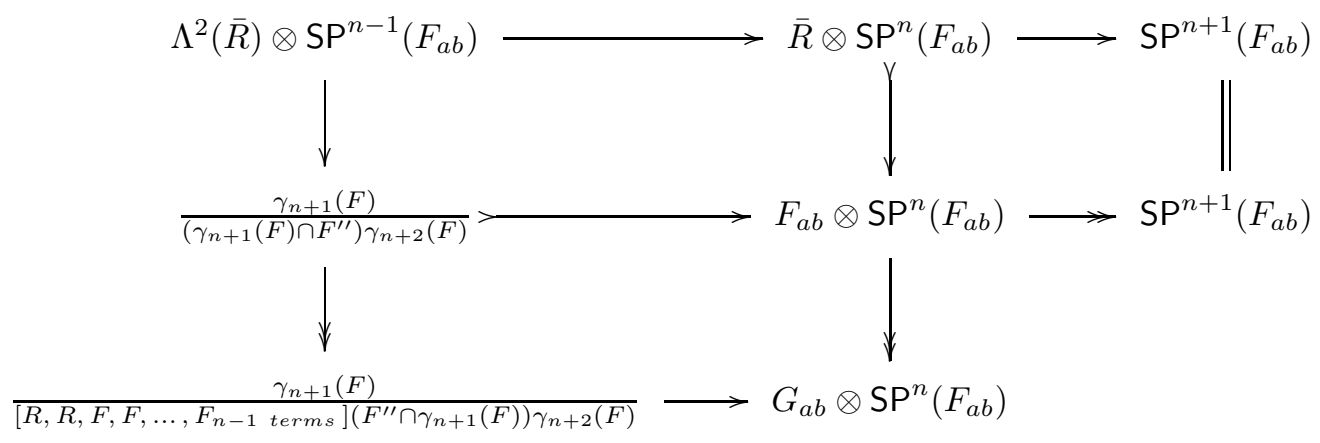

yields the natural exact sequence

$$
\begin{array}{r}
0 \rightarrow L_{1} \mathrm{SP}^{n+1}\left(G_{a b}\right) \rightarrow \frac{\gamma_{n+1}(F)}{[R, R, \underbrace{F, F, \ldots, F}_{n-1}]\left(F^{\prime \prime} \cap \gamma_{n+1}(F)\right) \gamma_{n+2}(F)} \rightarrow \\
G_{a b} \otimes \operatorname{SP}^{n}\left(F_{a b}\right) \rightarrow \operatorname{SP}^{n+1}\left(G_{a b}\right) \rightarrow 0,
\end{array}
$$

where $F^{\prime \prime}$ is the second derived subgroup of $F$.

\section{Identification theorems}

Theorem 4.1. Let $R$ and $S$ be normal subgroups of a free group $F$. Then there is a natural isomorphism

$$
\frac{F \cap\left(1+\mathbf{f}^{2} \mathbf{r}+\mathbf{s r}\right)}{\gamma_{2}\left(R \cap\left(F^{\prime} S\right)\right) \gamma_{3}(R)} \cong L_{1} \mathrm{SP}^{2}\left(\frac{R}{R \cap\left(F^{\prime} S\right)}\right) .
$$

Moreover, if $S$ is a normal subgroup of $R$, then

$$
\begin{aligned}
& \frac{F \cap(1+\mathbf{r f r}+\mathbf{s r})}{\gamma_{3}(R) S^{\prime}} \cong L_{1} \mathrm{SP}^{2}\left(\frac{R}{S R^{\prime}}\right), \\
& \frac{F \cap\left(1+\mathbf{r}^{2} \mathbf{f r}+\mathbf{s}^{2} \mathbf{r}\right)}{\gamma_{4}(R) \gamma_{3}(S)} \cong L_{2} \mathfrak{L}_{s}^{3}\left(\frac{R}{S R^{\prime}}\right) .
\end{aligned}
$$


Proof of 4.1 . Let us set

$$
Q:=R / R^{\prime}=R_{a b} \cong \frac{\mathbf{r}}{\mathbf{f r}}, \quad U:=\frac{R \cap\left(S F^{\prime}\right)}{R^{\prime}} .
$$

Observe that there is a natural monomorphism

$$
\frac{R}{R \cap\left(S F^{\prime}\right)} \otimes R_{a b} \hookrightarrow \frac{\mathbf{f}}{\mathbf{s}+\mathbf{f}^{2}} \otimes \frac{\mathbf{r}}{\mathbf{f r}} \cong \frac{\mathbf{f r}}{\mathbf{s r}+\mathbf{f}^{2} \mathbf{r}}
$$

resulting from the well-known identification of the second dimension subgroup:

$$
F \cap\left(1+\mathbf{s}+\mathbf{f}^{2}\right)=S F^{\prime} .
$$

Next observe that

$$
\Lambda^{2}(Q) / \Lambda^{2}(U) \cong \frac{R^{\prime}}{\gamma_{2}\left(R \cap\left(S F^{\prime}\right)\right) \gamma_{3}(F)}
$$

and

$$
F \cap\left(1+\mathbf{s r}+\mathbf{f}^{2} \mathbf{r}\right) \subseteq R^{\prime},
$$

since $\mathbf{s r}+\mathbf{f}^{2} \mathbf{r} \subset \mathbf{f r}$ and $F \cap(1+\mathbf{f r})=R^{\prime}$. Thus (4.1) follows from (3.2).

Proof of 4.2). Let us set

$$
Q:=R / R^{\prime}=R_{a b} \cong \frac{\mathbf{r}}{\mathbf{f r}}, \quad U:=\frac{S R^{\prime}}{R^{\prime}}
$$

Then

$$
\begin{aligned}
& \Lambda^{2}(Q) / \Lambda^{2}(U) \cong \frac{R^{\prime}}{S^{\prime} \gamma_{3}(R)} \\
& Q / U \otimes Q \cong \frac{\mathbf{r}}{\mathbf{s}+\mathbf{r f}} \otimes \frac{\mathbf{r}}{\mathbf{f r}} \cong \frac{\mathbf{r}^{2}}{\mathbf{s r}+\mathbf{r f r}} .
\end{aligned}
$$

Since $F \cap(1+\mathbf{s r}+\mathbf{r f r}) \subseteq R^{\prime}$, the isomorphism (4.2) follows from (3.2).

Proof of (4.3). In order to prove (4.3), observe that

$$
\begin{aligned}
& \mathcal{L}^{3}(Q) / \mathcal{L}^{3}(U) \cong \frac{\gamma_{3}(R)}{\gamma_{3}(S) \gamma_{4}(R)} \\
& \frac{Q \otimes Q}{U \otimes U} \otimes Q \cong \frac{\mathbf{r}^{2}}{\mathbf{s}^{2}+\mathbf{r}^{2} \mathbf{f}} \otimes \frac{\mathbf{r}}{\mathbf{f r}} \cong \frac{\mathbf{r}^{3}}{\mathbf{s}^{2} \mathbf{r}+\mathbf{r}^{2} \mathbf{f r}} .
\end{aligned}
$$

Since $F \cap\left(1+\mathbf{s}^{2} \mathbf{r}+\mathbf{r}^{2} \mathbf{f r}\right) \subseteq F \cap\left(1+\mathbf{r}^{2} \mathbf{f}\right)=\gamma_{3}(R)$, the isomorphism (4.3) follows from the exact sequence (3.5).

We next exhibit certain quotients constructed from a free presentation

$$
1 \rightarrow R \rightarrow F \rightarrow G \rightarrow 1
$$

of a group $G$ which are independent of the chosen free presentation, and in fact depend only on the second integral homology group $H_{2}(G)$. 
Theorem 4.2. Let $1 \rightarrow R \rightarrow F \rightarrow G \rightarrow 1$ be a free presentation of a group $G$, and let $S=[R, F]$. Then there are natural isomorphisms

$$
\begin{aligned}
& \frac{F \cap(1+\mathbf{r f r}+\mathbf{s r})}{\gamma_{2}(S) \gamma_{3}(R)} \cong L_{1} \mathrm{SP}^{2}\left(H_{2}(G)\right) \\
& \frac{F \cap\left(1+\mathbf{r f r}+\mathbf{r}^{2} \mathbf{f}\right)}{\gamma_{2}(S) \gamma_{3}(R)} \cong L_{1} \mathrm{SP}^{2}\left(H_{2}(G)\right) \\
& \frac{F \cap\left(1+\mathbf{s}^{2} \mathbf{r}+\mathbf{r}^{2} \mathbf{f r}\right)}{\gamma_{3}(S) \gamma_{4}(R)} \cong L_{2} \mathfrak{L}_{s}^{3}\left(H_{2}(G)\right)
\end{aligned}
$$

Proof of 4.4 and 4.5. Let us set

$$
Q:=R / R^{\prime}=R_{a b} \cong \frac{\mathbf{r}}{\mathbf{f r}}, \quad U:=S / R^{\prime}
$$

Then

$$
Q / U \otimes Q \cong R / S \otimes \frac{\mathbf{r}}{\mathbf{f r}} \cong \frac{\mathbf{r}}{(S-1)+\mathbf{r f}} \otimes \frac{\mathbf{r}}{\mathbf{f r}} \cong \frac{\mathbf{r}^{2}}{(S-1) \mathbf{r}+\mathbf{r f r}}
$$

and, since $F \cap(1+\mathbf{f r}+\mathbf{r f})=S$,

$$
Q / U \otimes Q \hookrightarrow \frac{\mathbf{r}}{\mathbf{f r}+\mathbf{r f}} \otimes \frac{\mathbf{r}}{\mathbf{f r}} \cong \frac{\mathbf{r}^{2}}{\mathbf{f r}^{2}+\mathbf{r f r}} .
$$

The exact sequence (3.2) implies that the left hand quotients in (4.4) and (4.5) are naturally isomorphic to $L_{1} \mathrm{SP}^{2}(R / S)$. Note that there is a natural isomorphism

$$
L_{1} \mathrm{SP}^{2}(R / S) \cong L_{1} \mathrm{SP}^{2}\left(H_{2}(G)\right) .
$$

To see this, observe that

$$
R / S \cong H_{2}(G) \oplus \mathcal{F}
$$

with $\mathcal{F}$ a free abelian group, and the asserted statements (4.4) and (4.5) thus follow from the cross-effect formula for the functor $L_{1} S^{2}$ (see, for example, (10.3) [2]):

$$
L_{1} \mathrm{SP}^{2}(A \oplus B)=L_{1} \mathrm{SP}^{2}(A) \oplus L_{1} \mathrm{SP}^{2}(B) \oplus \operatorname{Tor}(A, B) .
$$

To prove (4.6), observe that the sequence 3.5] implies that there is an exact sequence

$$
0 \rightarrow L_{2} \mathfrak{L}_{s}^{3}(R / S) \rightarrow \frac{\gamma_{3}(R)}{\gamma_{3}(S) \gamma_{4}(R)} \rightarrow \frac{\mathbf{r}^{2}}{\mathbf{r}^{2} \mathbf{f}+\mathbf{s}^{2}} \otimes \frac{\mathbf{r}}{\mathbf{f r}}
$$

Thus the isomorphism (4.6) follows from the natural isomorphisms

$$
L_{2} \mathfrak{L}_{s}^{3}(R / S)=L_{2} \mathfrak{L}_{s}^{3}\left(H_{2}(G)\right)
$$

and

$$
\frac{\mathbf{r}^{2}}{\mathbf{r}^{2} \mathbf{f}+\mathbf{s}^{2}} \otimes \frac{\mathbf{r}}{\mathbf{f r}}=\frac{\mathbf{r}^{3}}{\mathbf{r}^{2} \mathbf{f r}+\mathbf{s}^{2} \mathbf{r}}
$$


Using an implication, on the torsion in $L_{1} \mathrm{SP}^{2}\left(H_{2}(G)\right)$, of the result of R. Stöhr [12] (see also Yu. V. Kuzmin [9]) on the torsion in $F /\left[\gamma_{c}(R), F\right]$, we immediately have the following result.

Corollary 4.3. If $R$ is a norml subgroup of a free group $F, c \geq 2$ an integer, and $S=\gamma_{c}(R)$, then

$$
\frac{F \cap(1+\mathbf{s f s}+([S, F]-1) \mathbf{s})}{\gamma_{2}([S, F]) \gamma_{3}(S)}
$$

is a torsion group of exponent dividing $c^{2}$.

Theorem 4.4. If $R$ and $S$ are normal subgroups of a free group $F$ with $S$ normal in $R$, then there is a natural isomorphism

$$
\frac{F \cap\left(1+\mathbf{r f}+\mathbf{f s}+\mathbf{f}^{3}\right)}{[F, S] R^{\prime} \gamma_{3}(F)} \cong \operatorname{Coker}\left\{\operatorname{Tor}\left((F / R)_{a b},(F / S)_{a b}\right) \rightarrow L_{1} \mathrm{SP}^{2}\left((F / R)_{a b}\right)\right\} .
$$

Proof. Let us set

$$
E:=F / S F^{\prime}=(F / S)_{a b}, \quad I:=R F^{\prime} / S F^{\prime} .
$$

We then have natural isomorphisms

$$
\Lambda^{2}(E) \cong=\frac{\gamma_{2}(F)}{[F, S] \gamma_{3}(F)}, \quad \Lambda^{2}(E) / \Lambda^{2}(I) \cong \frac{\gamma_{2}(F)}{[F, S] R^{\prime} \gamma_{3}(F)},
$$

and

$$
E / I \otimes E \cong \frac{\mathbf{f}}{\mathbf{r}+\mathbf{f}^{2}} \otimes \frac{\mathbf{f}}{\mathbf{s}+\mathbf{f}^{2}} \cong \frac{\mathbf{f}^{2}}{\mathbf{r f}+\mathbf{f s}+\mathbf{f}^{3}} .
$$

The assertion in the theorem thus follows from Theorem 3.2

Theorem 4.5. If $1 \rightarrow R \rightarrow F \rightarrow G \rightarrow 1$ is a free presentation of a group $G$, and $n \geq 2$ an integer, then there is a natural isomorphism

$$
\frac{F \cap\left(1+\mathbf{f}\left(\left(F^{\prime}-1\right) \mathbb{Z}[F] \cap \mathbf{f}^{n}\right)+\mathbf{r f}^{n}+\mathbf{f}^{n+2}\right)}{[R, R, \underbrace{F, \ldots, F}_{n-1}]\left(F^{\prime \prime} \cap \gamma_{n+1}(F)\right) \gamma_{n+2}(F)} \cong L_{1} \mathrm{SP}^{n+1}\left(G_{a b}\right) .
$$

Proof. By (2.1), there is a natural isomorphism

$$
\begin{aligned}
(F / R)_{a b} \otimes \mathrm{SP}^{n}\left(F_{a b}\right) \cong \frac{\mathbf{f}}{\mathbf{r}+\mathbf{f}^{2}} \otimes \frac{\mathbf{f}^{n}}{\left(F^{\prime}-1\right) \mathbb{Z}[F] \cap \mathbf{f}^{n}+\mathbf{f}^{n+1}} \cong \\
\frac{\mathbf{f}^{n+1}}{\mathbf{r f} \mathbf{f}^{n}+\mathbf{f}\left(\left(F^{\prime}-1\right) \mathbb{Z}[F] \cap \mathbf{f}^{n}\right)+\mathbf{f}^{n+2}}
\end{aligned}
$$

The asserted statement thus follows from the sequence (3.6). 
In particular, taking $n=2,3$, the preceding theorem yields the following interesting result.

Corollary 4.6. There are natural isomorphisms

$$
\begin{aligned}
& \frac{F \cap\left(1+\mathbf{f}\left(F^{\prime}-1\right)+\mathbf{r} \mathbf{f}^{2}+\mathbf{f}^{4}\right)}{[R, R, F] \gamma_{4}(F)} \cong L_{1} \mathrm{SP}^{3}\left(G_{a b}\right) . \\
& \frac{F \cap\left(1+\mathbf{f}\left(\left(F^{\prime}-1\right) \mathbb{Z}[F] \cap \mathbf{f}^{3}\right)+\mathbf{r f}^{3}+\mathbf{f}^{5}\right)}{[R, R, F, F] F^{\prime \prime} \gamma_{5}(F)} \cong L_{1} \operatorname{SP}^{4}\left(G_{a b}\right) .
\end{aligned}
$$

Theorem 4.7. Let $R$ and $S$ be normal subgroups of a free group $F$. Then there is a natural isomorphism

$$
\frac{F \cap\left(1+\mathbf{f}^{2} \mathbf{r}^{2}+\mathbf{f}\left(R^{\prime}-1\right)+\mathbf{s r}^{2}\right)}{\left[R \cap\left(S F^{\prime}\right), R \cap\left(S F^{\prime}\right), R\right] \gamma_{4}(R)} \cong L_{1} \mathrm{SP}^{3}\left(\frac{R}{R \cap\left(S F^{\prime}\right)}\right)
$$

Proof. The sequence (3.6) implies that the kernel of the natural map

$$
\frac{\gamma_{3}(R)}{\left[R \cap\left(S F^{\prime}\right), R \cap\left(S F^{\prime}\right), R\right] \gamma_{4}(R)} \rightarrow \frac{R}{R \cap\left(S F^{\prime}\right)} \otimes \operatorname{SP}^{2}\left(R_{a b}\right)
$$

is $L_{1} \mathrm{SP}^{3}\left(\frac{R}{R \cap\left(S F^{\prime}\right)}\right)$. Thus the asserted statement follows from the natural embedding

$$
\frac{R}{R \cap\left(S F^{\prime}\right)} \otimes \mathrm{SP}^{2}\left(R_{a b}\right) \hookrightarrow \frac{\mathbf{f}}{\mathbf{s}+\mathbf{f}^{2}} \otimes \frac{\mathbf{r}^{2}}{\left(R^{\prime}-1\right)+\mathbf{f r}^{2}} \cong \frac{\mathbf{f r}^{2}}{\mathbf{s r}^{2}+\mathbf{f}^{2} \mathbf{r}^{2}+\mathbf{f}\left(R^{\prime}-1\right)}
$$

Theorem 4.8. If $1 \rightarrow R \rightarrow f \rightarrow G \rightarrow 1$ is a free presentation of a group $G$, then

$$
F \cap\left(1+\mathbf{f}^{2} \mathbf{r}^{2}+\mathbf{f}\left(R^{\prime}-1\right)\right)=\left[R \cap F^{\prime}, R \cap F^{\prime}, R\right] \gamma_{4}(R),
$$

and there is a natural isomorphism

$$
\frac{F \cap\left(1+\mathbf{r f r}^{2}+\mathbf{f r}^{3}+\mathbf{r}\left(R^{\prime}-1\right)\right)}{[[R, F],[R, F], R] \gamma_{4}(R)} \cong L_{1} \mathrm{SP}^{3}\left(H_{2}(G)\right) .
$$

Proof. Since $R /\left(R \cap F^{\prime}\right)$ is torsion-free, $L_{1} \mathrm{SP}^{3}\left(R /\left(R \cap F^{\prime}\right)\right)=0$ and the sequence (3.6) implies that there is a natural monomorphism

$$
\frac{\gamma_{3}(R)}{\left[R \cap F^{\prime}, R \cap F^{\prime}, R\right] \gamma_{4}(R)} \hookrightarrow R /\left(R \cap F^{\prime}\right) \otimes \operatorname{SP}^{2}\left(R_{a b}\right)
$$

The identification (4.9) follows from the natural monomorphism

$$
R /\left(R \cap F^{\prime}\right) \otimes \mathrm{SP}^{2}\left(R_{a b}\right) \hookrightarrow \frac{\mathbf{f}}{\mathbf{f}^{2}} \otimes \frac{\mathbf{r}^{2}}{\left(R^{\prime}-1\right)+\mathbf{f r}^{2}} \cong \frac{\mathbf{f r}^{2}}{\mathbf{f}^{2} \mathbf{r}^{2}+\mathbf{f}\left(R^{\prime}-1\right)}
$$


To prove (4.10), we first observe that there exists an exact sequence

$$
0 \rightarrow L_{1} \mathrm{SP}^{3}(R /[R, F]) \rightarrow \frac{\gamma_{3}(R)}{[[R, F],[R, F], R] \gamma_{4}(R)} \rightarrow R /[R, F] \otimes \mathrm{SP}^{2}\left(R_{a b}\right) .
$$

Therefore the assertion follows from the isomorphisms

$$
R /[R, F] \otimes \mathrm{SP}^{2}\left(R_{a b}\right) \cong \frac{\mathbf{r}}{\mathbf{r f}+\mathbf{f r}} \otimes \frac{\mathbf{r}^{2}}{\left(R^{\prime}-1\right)+\mathbf{f r}^{2}} \cong \frac{\mathbf{r}^{3}}{\mathbf{r}\left(R^{\prime}-1\right)+\mathbf{r f r}^{2}+\mathbf{f r}^{3}}
$$

and the fact that $L_{1} \mathrm{SP}^{3}(R /[R, F])=L_{1} \mathrm{SP}^{3}\left(H_{2}(G)\right)$.

\section{Limits}

A theory of limits for functors on the category of free presentation of groups is developed in [7, [6], 11]. For a group $G$, consider the category $\mathcal{E}$ of free presentations

$$
1 \rightarrow R \rightarrow F \rightarrow G \rightarrow 1 .
$$

For any functor (also called a representation) $\mathcal{F}: \mathcal{E} \mapsto \mathcal{A}$, its limit $\lim _{\leftarrow} \mathcal{F}$ presents a well defined functor from the category of groups to $\mathcal{A}$. To illustrate, let us recall one example from [11]. As mentioned in the introduction, for a polynomial functor of degree $n$, the intermediate derived functors (from the first till the $(n-2)$ nd) as a rule have a complicated nature. In [11, the authors obtained a limit formula for such a functor, namely, $L_{1} \mathrm{SP}^{3}$ :

$$
L_{1} \mathrm{SP}^{3}\left(G_{a b}\right)=\lim _{\longleftarrow} \frac{\gamma_{2}(F)}{\left[R^{\prime}, F\right] \gamma_{3}(F)} .
$$

In the present work, we make further contribution to the theory of limits, with the help of the results obtained above.

There are two basic simple properties of limits which we will use (see [7]):

1) The inverse limit is left exact; for an exact sequence of representations

$$
\mathcal{F} \hookrightarrow \mathcal{G} \rightarrow \mathcal{H}
$$

there is a natural exact sequence of limits

$$
\lim _{\longleftarrow} \mathcal{F} \hookrightarrow \lim _{\longleftarrow} \mathcal{G} \rightarrow \lim _{\longleftarrow} \mathcal{H}
$$

2) For any representations $\mathcal{F}, \mathcal{G}$,

$$
\lim _{\leftarrow} \mathcal{F} \otimes \mathcal{G}\left(F_{a b}\right)=0 ;
$$

i.e, if a representation is the tensor product of some representation with a functor which depends only on $F_{a b}$, its limit is zero. 
Theorem 5.1.

$$
\begin{aligned}
& \lim _{\longleftarrow} \frac{R^{\prime}}{\gamma_{2}([R, F]) \gamma_{3}(R)} \cong L_{1} \mathrm{SP}^{2}\left(H_{2}(G)\right), \\
& \lim _{\longleftarrow} \frac{\gamma_{3}(R)}{\left[\gamma_{2}\left(R \cap F^{\prime}\right), R\right] \gamma_{4}(R)}=0, \\
& \lim _{\longleftarrow} \frac{\gamma_{3}(R)}{\left[\gamma_{2}([R, F]), R\right] \gamma_{4}(R)} \cong L_{1} \mathrm{SP}^{3}\left(H_{2}(G)\right), \\
& \lim _{\longleftarrow} \frac{\gamma_{3}(R)}{\gamma_{3}([R, F]) \gamma_{4}(R)} \cong L_{2} \mathfrak{L}_{s}^{3}\left(H_{2}(G)\right), \\
& \underset{\leftarrow}{\lim } \frac{\gamma_{4}(F)}{[R, R, F, F] F^{\prime \prime} \gamma_{5}(F)} \cong L_{1} \mathrm{SP}^{4}\left(G_{a b}\right), \\
& \lim _{\longleftarrow} \frac{\gamma_{4}(R)}{[[R, F],[R, F], R, R] R^{\prime \prime} \gamma_{5}(R)} \cong L_{1} \mathrm{SP}^{4}\left(H_{2}(G)\right), \\
& \lim _{\longleftarrow} \frac{R^{\prime \prime}}{\gamma_{2}([R, R, F]) \gamma_{3}\left(R^{\prime}\right)} \cong L_{1} \mathrm{SP}^{2}\left(H_{2}\left(G, \mathrm{SP}^{2}(\mathbf{g})\right)\right),
\end{aligned}
$$

in particular, if $G$ is 2-torsion-free, then

$$
\lim _{\longleftarrow} \frac{R^{\prime \prime}}{\gamma_{2}([R, R, F]) \gamma_{3}\left(R^{\prime}\right)} \cong L_{1} \operatorname{SP}^{2}\left(H_{4}(G, \mathbb{Z} / 2)\right) .
$$

Proof. It is shown in the proof of Theorem 4.2 that there is the following exact sequence

$$
0 \rightarrow L_{1} \mathrm{SP}^{2}\left(H_{2}(G)\right) \rightarrow \frac{R^{\prime}}{\gamma_{2}([R, F]) \gamma_{3}(R)} \rightarrow R /[R, F] \otimes R_{a b}
$$

The Magnus embedding $R_{a b} \hookrightarrow \mathbb{Z}[G] \otimes F_{a b} \rightarrow \mathbf{g}$ implies that

$$
\lim _{\longleftarrow} R /[R, F] \otimes R_{a b} \hookrightarrow \lim _{\longleftarrow} R /[R, F] \otimes \mathbb{Z}[G] \otimes F_{a b}=0
$$

The isomorphism (5.2) thus follows.

The monomorphism (4.11) implies that there is a monomorphism

$$
\frac{\gamma_{3}(R)}{\left[R \cap F^{\prime}, R \cap F^{\prime}, R\right] \gamma_{4}(R)} \hookrightarrow F_{a b} \otimes \mathrm{SP}^{2}\left(R_{a b}\right),
$$

and so the statement (5.3) follows.

The sequence (4.13) implies that there is the following exact sequence

$$
0 \rightarrow L_{1} \mathrm{SP}^{3}\left(H_{2}(G)\right) \rightarrow \frac{\gamma_{3}(R)}{[[R, F],[R, F], R] \gamma_{4}(R)} \rightarrow R /[R, F] \otimes \mathrm{SP}^{2}\left(F_{a b}\right)
$$

and the isomorphism (5.4) follows. 
The same arguments show that the sequence (4.7) implies (5.5). Further, the sequence (3.6) implies (5.6). The sequence (3.6) implies that there is the following exact sequence

$$
0 \rightarrow L_{1} \mathrm{SP}^{4}(R /[R, F]) \rightarrow \frac{\gamma_{4}(R)}{[[R, F],[R, F], R, R] R^{\prime \prime} \gamma_{5}(R)} \rightarrow R /[R, F] \otimes \operatorname{SP}^{3}\left(R_{a b}\right)
$$

Since

$$
\lim _{\longleftarrow} R /[R, F] \otimes \mathrm{SP}^{3}\left(R_{a b}\right) \hookrightarrow \lim _{\longleftarrow} R /[R, F] \otimes \mathrm{SP}^{3}\left(F_{a b}\right)=0,
$$

the isomorphism (5.7) follows. The isomorphism (5.8) follows in the same way. To see it, we get first the isomorphism

$$
L_{1} \mathrm{SP}^{2}\left(R^{\prime} /[R, R, F]\right) \cong \lim _{\longleftarrow} \frac{R^{\prime \prime}}{\gamma_{2}([R, R, F]) \gamma_{3}\left(R^{\prime}\right)}
$$

Now the identifications (5.8) and (5.1) follow from the results of R. Stöhr [12], which describe the torsion of $R^{\prime \prime} /\left[R^{\prime \prime}, F\right]$.

We end the paper with a problem. A detailed analysis analogous to that done in 11. shows that, for any $n \geq 2$, the limit

$$
\lim _{\longleftarrow} \frac{\gamma_{n}(F)}{\gamma_{n}(R) \gamma_{n+1}(F)}
$$

can be identified with the $(n-1)$ st derived functor of the $n$th super-Lie power of $G_{a b}$. On the other hand,

$$
\lim _{\longleftarrow} \frac{\gamma_{n}(F)}{[R, \underbrace{F, F, \ldots, F}_{n-1}] \gamma_{n+1}(F)} \cong \mathcal{L}^{n}\left(G_{a b}\right) .
$$

The identification (5.1) suggests a conjecture that the limit

$$
\lim _{\longleftarrow} \frac{\gamma_{n}(F)}{[\underbrace{R, \ldots, R}_{k}, \underbrace{F, \ldots, F}_{n-k}] \gamma_{n+1}(F)}
$$

may be related to the $(k-1)$ st derived functor of some well-described polynomial functor of degree $n$ applied to $G_{a b}$.

Problem. Describe the functors (5.9) for all $n>3$ and $k=2, \ldots, n-1$.

\section{References}

[1] L. Breen and R. Mikhailov: Derived functors of non-additive functors and homotopy theory, Algebr. Geom. Topol., 11 (2011), 327 - 415. 
[2] A. Dold and D. Puppe: Homologie nicht additiver Funktoren Anwendugen, Annales de l'institut Fourier, tome 11, nº 6 (1961), 201 - 312.

[3] S. Eilenberg and S. Mac Lane, On the groups H(П,n) II, Ann. of Math. (2) 60 (1954) 49-139.

[4] Narain Gupta: Free Group Rings, Contemporary Mathematics, Vol. 66, American Mathematical Society, 1987.

[5] M. Hartl, R. Mikhailov and I. B. S. Passi: Dimension quotients, J. Indian Math. Soc., New Ser. Spec. Centenary Vol., 63-107 (2007).

[6] Sergei O. Ivanov and Roman Mikhailov: Higher limits, homology theories and frcodes, arXiv:1510.09044v1 [math.GR].

[7] Sergei O. Ivanov and Roman Mikhailov: A higher limit approach to homology theories, J. Pure and Appl. Algebra 219 (2015), 1915-1939.

[8] B. Köck: Computing the homology of Koszul complexes, Trans. Amer. Math. Soc., 353 (2001), 3115 - 3147.

[9] Yu. V. Kuzmin: On elements of finite order in free groups of some varieties. Mat. Sb. 119, 119- 131 (1982) [Russian].

[10] Roman Mikhailov and Inder Bir S. Passi: Lower Central and Dimension Series of Groups, LNM Vol. 1952, Springer 2009.

[11] Roman Mikhailov and Inder Bir S. Passi: Generalized dimension subgroups and derived functors, J. Pure Appl. Algebra 220 (2016), 2143-2163.

[12] R. Stöhr: On Gupta representations of central extensions, Math. Z. 187, 259-267 (1984).

[13] L. R. Vermani: On subgroups determined by ideals of an integral group ring, Passi, I. B. S. (ed.), Algebra. Some recent advances. Basel: Birkhauser. Trends in Mathematics. 227-242 (1999).

Roman Mikhailov, St Petersburg Department of Steklov Mathematical Institute, and Chebyshev Laboratory, St Petersburg State University, 14th Line, 29b, Saint Petersburg 199178 Russia

E-mail: romanvm@mi.ras.ru

Inder Bir S. Passi, Centre for Advanced Study in Mathematics, Panjab University, Sector 14, Chandigarh 160014 India, and Indian Institute of Science Education and Research, Mohali (Punjab) 140306 India

E-mail: ibspassi@yahoo.co.in 